\title{
TBX2 Regulates Proliferation, Apoptosis, and Cholesterol Generation of Bovine Through Maintaining Mitochondrial Function and Autophagy in Bovine Cumulus Cell
}

\author{
Sheng-Peng Li \\ Jilin University \\ Wen-Yin Xie \\ Jilin University \\ Wen-Jie Yu \\ Jilin University \\ Yan-Xia Peng
}

Jilin University

Chang Liu

Jilin Business and Technology College

Zi-Bin Liu

Jilin University

Xiao-Shi Cai

Jilin University

Cheng-Zhen Chen

Jilin University

\section{Bao Yuan}

Jilin University

Nam-Hyung Kim

Jilin University

Hao Jiang ( $\sim$ jhhaojiang@jlu.edu.cn )

Jilin University

Jia-Bao Zhang

Jilin University

\section{Research Article}

Keywords: TBX2, Cumulus cells, ROS, Autophagy, Physiological function

Posted Date: March 23rd, 2021 
DOl: https://doi.org/10.21203/rs.3.rs-333712/v1

License: (c) (1) This work is licensed under a Creative Commons Attribution 4.0 International License. Read Full License 


\title{
$T B X 2$ regulates proliferation, apoptosis, and cholesterol generation of bovine through maintaining mitochondrial function and autophagy in bovine cumulus cell
}

Sheng-Peng Li ${ }^{1, *}$, Wen-Yin Xie ${ }^{1, *}$, Wen-Jie Yu ${ }^{1}$, Yan-Xia Peng ${ }^{1}$, Chang Liu ${ }^{2}, \mathrm{Zi-Bin}$ Liu $^{1}$, Xiao-Shi Cai ${ }^{1}$, Cheng-Zhen Chen ${ }^{1}$, Bao Yuan ${ }^{1}$, Nam-Hyung Kim ${ }^{1,3}$, Hao Jiang ${ }^{1, \S}$, Jia-Bao Zhang ${ }^{1, \S}$

${ }^{1}$ Department of Laboratory Animals, Jilin Provincial Key Laboratory of Animal Model, Jilin University, Changchun, Jilin, 130062, People's Republic of China

${ }^{2}$ School of Grains, Jilin Business and Technology College, Changchun, 130507, China ${ }^{3}$ Department of Animal Science, Chungbuk National University, Cheongju, Chungbuk, 28644, Republic of Korea

*Sheng-Peng Li, Wen-Yin Xie contributed equally to this work.

${ }^{\S}$ Corresponding authors at:

Department of Laboratory Animals, Jilin Provincial Key Laboratory of Animal Model, Jilin University, Changchun, Jilin, 130062, People's Republic of China.

E-mail addresses: jhhaojiang@jlu.edu.cn (H. Jiang); zjb@jlu.edu.cn (J. Zhang).

\begin{abstract}
$T B X 2$ is a member of T-box gene family whose members are highly conserved in evolution and encoding genes are involved in the regulation of developmental processes. The encoding genes play an important role in growth and development, but its regulatory effects in bovine cumulus cells are still unclear. In this study, the changes of cell physiological function were detected after $T B X 2$ gene was knocked down. Compared with the control group, when $T B X 2$ was inhibited, the levels of autophagy, apoptosis and reactive oxygen species in cumulus cells were increased, the proliferation, expansion ability and the amount of cholesterol secreted by cells were significantly decreased, and the cell cycle was also disrupted. The results showed that TBX2 could regulate the normal physiological activities of bovine cumulus cells.
\end{abstract}

Keywords: TBX2, Cumulus cells, ROS, Autophagy, Physiological function

\section{Abbreviations:}

$T B X 2$, T-box transcription factor 2; $q P C R$, quantitative polymerase chain reaction; $F I T C$, fluorescein isothiocyanate; $P I$, propidium iodide; $B C L 2$, B-cell lymphoma 2; $B A X$, BCL2 associated $\mathrm{X} ; L C 3 B$, light chain 3 beta; $R O S$, reactive oxygen species; $P T X 3$, Pentraxin 3; HAS2, Hyaluronan synthase 2; PTGS2, Prostaglandin-endoperoxide synthase 2; $C D K 1$, Cyclin dependent kinase 1; CDK2, Cyclin dependent kinase 2; $C D K 4$, Cyclin dependent kinase 4; CDK6, Cyclin dependent kinase 6; ERK, extracellular signal-regulated kinase; COCs, cumulus-oocyte complexes; PVA, polyvinyl alcohol; $M M P$, mitochondrial membrane potential; $J C-1,5,5^{\prime}, 6,6^{\prime}-$ tetrachloro-1,1',3,3'-tetraethyl-benzimidazolylcarbocyanine iodide; $D C F H-D A, 2^{\prime}, 7^{\prime}-$ 
dichlorofluorescein diacetate; $P B S$, phosphate buffered saline; TBST, Tris-buffered saline with $0.1 \%$ Tween ${ }^{\circledR} 20$ detergent; $S D$, standard deviation.

\section{Introduction}

Improving the quality of oocytes is important for animal husbandry and human fertility ${ }^{1}$ because the quality of oocytes is the primary factor affecting fertilization and breeding of healthy offspring ${ }^{2}$. As a subgroup of granular cells, cumulus cells play important role in the nutrition and maturation of oocytes ${ }^{3}$. Oocytes are coupled to surrounding cumulus cells through interstitial junctions ${ }^{4}$, and this highly specific membrane junction forms the regulatory system that mediates the intercellular transfer of metabolites and regulatory molecules ${ }^{5}$. Under normal physiological conditions, oocytes generate growth factors that regulate cumulus cells, and cumulus cells provide nutrients for oocyte growth through intracellular exchange; these processes are interdependent but closely related ${ }^{6}$. In addition, oocytes, under pathological conditions, protect themselves against oxidative stress through antioxidant-scavenging enzymatic (e.g., involving catalase and glutathione peroxidase) and nonenzymatic (e.g., involving ascorbic acid and reduced glutathione) networks provided by the surrounding cumulus cells $^{7-9}$.

As intracellular energy factories, mitochondria are the main producer of intracellular ROS ${ }^{10}$, which are involved in processes such as cell differentiation, cell signal transmission, cell apoptosis and the regulation of cell growth and the cell cycle. When excessive production of ROS occurs, the accumulation of oxidants exceeds the cell's ability to clear them, and the oxidation system and antioxidant system become unbalanced. A sharp increase in ROS will lead to oxidative stress, causing cell damage and apoptosis ${ }^{11}$. In the process of cell apoptosis, the ratio of $B A X / B C L 2{ }^{12,13}$ directly determines the degree of opening of various channels in the mitochondrial outer membrane, and $B A X$ and $B C L 2$ represent a regulatory hub of cell apoptosis, while caspase 3 usually co-regulates apoptosis with $B A X$ and $B C L 2^{14}$. In addition, preferential autophagy of damaged or excess organelles such as peroxisomes, the endoplasmic reticulum, and mitochondria can occur in response to ROS ${ }^{15}$. ROSmediated autophagy and apoptosis of cumulus cells will affect their secretory functions, thus potentially affecting the development and quality of oocytes ${ }^{16-18}$.

T-box gene family is a phylogenetically conserved family of genes that share a common DNA-binding domain ${ }^{19}$ and is important in the regulation of body development. Research has shown that $T B X 2$ functions as a transcriptional repressor during germ layer formation, and this activity is mediated in part through repression of target genes stimulated in the mesendoderm by transactivating T-box proteins ${ }^{20}$. In addition, $T B X 2$, as a transcription factor, is involved in embryonic development and cell cycle regulation and inhibits the cycle regulation factors $p 21$ and $p 14$ to make cells resist senescence ${ }^{21-23}$. Recent research also showed that after the TBX2 gene was knocked down, cell proliferation and invasion were significantly decreased; after TBX2 was overexpressed, cyclin E and the phosphorylated extracellular signal-regulated kinase levels were upregulated ${ }^{24}$.

Although $T B X 2$ has been extensively studied in cancer cells ${ }^{25}$, its biological functions in bovine cumulus cells remains unclear. This study investigated the effects 
of TBX2 on ROS levels, mitochondrial function, and cell proliferation in cumulus cells by inhibiting the expression of $T B X 2$. The results will provide a new basis for understanding the biological roles of $T B X 2$ as well as cumulus cells.

\section{Result}

Inhibition of TBX2 reduces cell proliferation and disrupts the cell cycle in bovine cumulus cells. Three pairs of $T B X 2$-specific siRNAs were designed. After comparison, the most effective and stable siRNA (siRNA-708) was selected for subsequent experiments (Supplementary Figure 1). As shown in Fig. 1A, the cell cycle was significantly changed after $T B X 2$ inhibition, and the proportion of G1 phase cells in the TBX2-inhibited group increased to $1.38 \pm 0.05$ times that in the control group $(\mathrm{P}<0.01)$. The proportion of $\mathrm{S}$ phase cells decreased to $0.51 \pm 0.04$ times that in the control group $(\mathrm{P}<0.01)$. The proportion of $\mathrm{G} 2$ cells increased to $1.76 \pm 0.07$ times that in the control group $(\mathrm{P}<0.01)$. The expression levels of $C D K 1$ and $C D K 4$ genes increased to $1.77 \pm 0.20(\mathrm{P}<0.01)$ and $2.09 \pm 0.23$ times $(\mathrm{P}<0.01)$ that in the control group, respectively. The $C D K 6$ gene was downregulated $0.64 \pm 0.11$ times $(\mathrm{P}<0.01)$, while the expression of the $C D K 2$ gene was upregulated $1.08 \pm 0.21$ times $(\mathrm{P}>0.01$, Fig. 1B). In addition, the cell index in the TBX2-inhibited group was lower than that of the control group after approximately $5 \mathrm{~h}$, and this effect lasted for at least $40 \mathrm{~h}$ (Fig. 1C). After $T B X 2$ inhibition, the apoptosis rate of cumulus cells increased from $12.48 \pm$ $2.50 \%$ to $19.61 \pm 1.95 \%$ (Fig. 1D). Compared with the control group, the BAX/BCL2 level in the $T B X 2$ inhibition group increased by $1.53 \pm 0.11$ times (Fig. 1E).

TBX2 inhibition leads to an increase in ROS accumulation in bovine cumulus cells. ROS are important factors that induce apoptosis in cells. Therefore, we tested whether $T B X 2$ regulates apoptosis by affecting intracellular ROS accumulation. As shown in Fig. $2 \mathrm{~A}$ and $\mathrm{B}$, the DCFH fluorescence levels in the $T B X 2$ inhibition group were $1.37 \pm 0.09$ fold higher than those in the si-NC group. Furthermore, flow cytometry analysis (Fig. 2C) indicated that the intracellular ROS levels of the TBX2 inhibition group were significantly increased to $1.46 \pm 0.12$ times $(\mathrm{P}<0.01)$ those of the si-NC group. These suggested that inhibition of TBX2 caused ROS stress in bovine cumulus cells.

Inhibition of TBX2 disrupts mitochondrial function. As shown in Fig. 3A and B, the $\Delta \Psi \mathrm{m}$ of the average cell was calculated as a ratio of red fluorescence intensity (Jaggregates; corresponding to activated mitochondria) to green fluorescence intensity (J-monomers; corresponding to inactive mitochondria). The results showed that after inhibition of $T B X 2$, the $\triangle \Psi \mathrm{m}$ decreased to $0.38 \pm 0.05$ times compared to those in the si-NC group $(\mathrm{P}<0.01)$. In addition, the ATP level (Fig. $3 \mathrm{C})$ in $T B X 2$-inhibited cumulus cells decreased to $0.56 \pm 0.04$ times compared to those in the si-NC group $(\mathrm{P}<0.01)$. These results suggested that the mitochondrial activity and function in cumulus cells decreased significantly after the inhibition of $T B X 2$.

Inhibition of TBX2 increases autophagy levels. Autophagy, which is usually 
measured by the levels of $L C 3 B$, maintains microenvironment stability in vivo, thereby reducing damage to the cells. After inhibition of $T B X 2$, the immunofluorescence results showed a significant increase in number of cytoplasmic $L C 3 B \operatorname{dot}$ (Fig. 4A). The Western blot results were also consistent with this finding. The relative protein levels of $L C 3 B$ in the $T B X 2$-inhibited group were $1.38 \pm 0.09$-fold higher than those in the siNC group $(\mathrm{P}<0.01$, Fig. 4B).

TBX2 inhibition prevents cumulus cell expansion. As shown in Fig. 5A and B, the total width of the scratches decreased with time. cumulus cells began to exhibit considerable expansion from $12 \mathrm{~h}$ to $36 \mathrm{~h}$. The relative expansion widths in the si-NC group were $12.43 \pm 3.45 \%, 39.58 \pm 3.52 \%$, and $80.54 \pm 3.98 \%$ at $12 \mathrm{~h}, 24 \mathrm{~h}$, and $36 \mathrm{~h}$, respectively, while the relative expansion widths in the $T B X 2$-inhibited group were $11.98 \pm 2.86 \%, 24.86 \pm 2.54 \%(\mathrm{P}<0.01)$, and $51.11 \pm 7.79 \%(\mathrm{P}<0.01)$ at $12 \mathrm{~h}, 24 \mathrm{~h}$, and $36 \mathrm{~h}$, respectively. Meanwhile, the mRNA expression levels of the cumulus cell expansion-related genes $P T G S 2, P T X 3$, and HAS2 were also significantly decreased by $0.72 \pm 0.14,0.43 \pm 0.08$, and $0.63 \pm 0.11$ times, respectively, in the si-TBX2 group compared with the si-NC group $(\mathrm{P}<0.01$, Fig. $5 \mathrm{C})$. In addition, relative cholesterol levels were significantly reduced by $0.58 \pm 0.02$-fold in the $T B X 2$ inhibition group compared with the si-NC group $(\mathrm{P}<0.01$. Fig. 5D).

\section{Discussion}

In this study, we inhibited the expression of the $T B X 2$ gene to explore the physiological role of $T B X 2$ in bovine cumulus cells. In general, after $T B X 2$ was inhibited, the cell cycle was disrupted, the intracellular oxidative stress and autophagy levels were increased, and the rate of cell apoptosis was also increased, suggesting that $T B X 2$ can regulate the physiological functions of bovine cumulus cells.

The cell cycle plays an important role in cell proliferation and apoptosis ${ }^{26,27}$. Previous studies had shown that inhibition of $T B X 2$ resulted in an increase in G1 phase cells and a decrease in $\mathrm{S}$ phase cells ${ }^{28,29}$. Changes in $C D K 2$ expression may regulate the G1/S phase transition, as well as DNA synthesis and replication in S phase ${ }^{30,31}$. However, the expression of $C D K 2$ did not change significantly. This may be because $T B X 2$ promoted cell cycle progression through Cyclin D1 and RB-E2F1 but not $p 21$ and $C D K 2^{29}$. The differential expression of $C D K 1$ indicates that inhibition of $T B X 2$ can promote entry into $M$ phase and the transition from $G 2$ to $M$ phase, thus contributing to mitotic progression in cell division ${ }^{32,33}$. In addition, inhibition of TBX2 may also be associated with D-type cyclins (D1, D2, and D3) and CDK4/6 and disrupt the essential processes for entry into $\mathrm{G} 1$ phase ${ }^{34}$. In the $\mathrm{G} 1$ phase, CDK4/6-cyclin D promotes cell cycle progression by means of retinoblastoma protein phosphorylation and sequestration of $p 21$ and $p 27$. This indicated that inhibition of TBX2 reduced the release of $C D K 2$-cyclin $E$ complexes as well as $C D K 2$ kinase activity ${ }^{35}$. In addition, these results are also consistent with existing reports and our subsequent findings that inhibition of $T B X 2$ affects cell proliferation. Changes in cell proliferative capacity are influenced by nutrient utilization, mitochondrial function and the physiological state of cells and ultimately affect the biological functions of cells ${ }^{36}$. Combined with the results 
of previous studies, our findings suggest that $T B X 2$ may have a potential regulatory effect on the physiological and secretory functions of cumulus cells ${ }^{37}$.

Apoptosis can also be initiated by decreased mitochondrial activity and ROSinduced oxidative stress in addition to abnormal changes in the cell cycle ${ }^{38}$. In this study, inhibition of $T B X 2$ significantly increased $B A X / B C L 2$ levels and the apoptosis rate. This is in accordance with the findings of another study in which $T B X 2$ overexpression reduced caspase 3 cleavage and induced BCL2 and p-Drp1 upregulation ${ }^{39}$. In addition, ROS accumulation is associated with mitochondrial fission and affects cell proliferation and apoptosis ${ }^{40-42}$. Given with the MMP and ROS assay results, we suspect that inhibition of $T B X 2$ disrupts the mitochondrial fission/fusion balance in cumulus cells ${ }^{28}$, which results in an increase in intracellular ROS levels and a decrease in mitochondrial function. Subsequently, the level of autophagy can be upregulated by cells to adapt to the adverse internal environment ${ }^{43}$, which indicates that the level of $T B X 2$ is important for maintaining environmental stability and generating the building blocks necessary for macromolecular synthesis, energy production, and cell survival ${ }^{44}$. physiological function of cumulus cells. Although the expansion of monolayer cells on a hard surface does not fully reflect cumulus cell expansion, we hypothesized that TBX2 would regulate the cumulus cell contact, as $T B X 2$ has been shown to downregulate key factors affecting cumulus cell expansion, such as PTX3, PTGS2, and HAS2 ${ }^{45,46}$. Previous studies showed that oocytes are unable to synthesize cholesterol and require cumulus cells to provide products of the cholesterol biosynthetic pathway ${ }^{47,48}$. The expansion of cumulus cells has a substantial regulatory effect on oocyte secretion, which is associated with metabolic processes in COCs, especially cholesterogenesis ${ }^{49,50}$. In this study, inhibition of $T B X 2$ led to a decrease in the cholesterol level, which indicates that inhibition of $T B X 2$ will lead to a decrease in precursor substances for the synthesis of steroid hormones ${ }^{51}$. This also suggests that $T B X 2$ may have a regulatory effect on the secretory function of cumulus cell and affect oocyte maturation and fertilization ${ }^{52,53}$.

\section{Conclusions}

In conclusion, the results of this study showed that $T B X 2$ has a regulatory role in maintaining the mitochondrial function and autophagy level of cumulus cells and in regulating the proliferation, apoptosis, and cholesterol generation of bovine cumulus cells, thus playing a potential role in oocyte development, maturation, and subsequent fertilization.

\section{Authors contributions}

Sheng-Peng Li, Wen-Yin Xie, Wen-Jie Yu, Yan-Xia Peng, Zi-Bin Liu, and Xiao-Shi Cai carried the experiments. Sheng-Peng Li, Chang Liu, Bao Yuan, and Cheng-Zhen Chen performed the data analyses. Sheng-Peng Li, Wen-Jie Yu, Yan-Xia Peng, and Chang Liu drew the images. Sheng-Peng Li, Xiao-Shi Cai, and Hao Jiang wrote and revised the manuscript. Hao Jiang, Nam-Hyung Kim, and Jia-Bao Zhang supervised the study. 


\section{Acknowledgments}

This study was supported by the National Natural Science Foundation of China (31972570), the Modern Agricultural Industry Technology System (CARS-37), and the Science and Technology Project of Jilin Province (SXGJSF2017-6).

\section{Declaration of Competing Interest}

The authors declare that they have no known competing financial interests or personal relationships that could have appeared to influence the work reported in this paper.

\section{Material and method}

The chemicals and reagents that we used in the experiment were bought from Sigma-Aldrich (St. Louis, MO, USA) except expressly stated elsewise in the article.

Isolation and culture of bovine cumulus cells. Bovine ovaries without corpus luteum were collected from a local slaughterhouse and transported to the laboratory at $35{ }^{\circ} \mathrm{C}$ in saline supplemented with $75 \mathrm{mg} / \mathrm{mL}$ penicillin $\mathrm{G}$ and $50 \mathrm{mg} / \mathrm{mL}$ streptomycin sulfate. COCs were aspirated from follicles 3 to $8 \mathrm{~mm}$ in diameter using a $10-\mathrm{mL}$ syringe with an 18-gauge needle. Then, COCs surrounded by a minimum of three cumulus cells were selected and washed three times in Tyrode's lactate HEPES (TLHEPES) supplemented with $0.1 \%$ (w/v) PVA and gentamycin $(0.05 \mathrm{~g} / \mathrm{L})$. Subsequently, COCs were dissociated with $1 \%$ hyaluronidase to separate cumulus cells and oocytes. After removing the oocytes, the cumulus cells were centrifuged, resuspended, and seeded into the culture plate with cell culture medium including DMEM/F12 (Gibco, Grand Island, NY, USA), 1\% penicillin and streptomycin (HyClone, Logan, UT, USA) and $10 \%$ fetal bovine serum (Biological Industries, Kibbutz Beit Haemek, Israel) and cultured at $37^{\circ} \mathrm{C}$ in $5 \% \mathrm{CO}_{2}$.

siRNA treatment. A total of $1 \times 10^{5}$ cells were seeded into six-well plates containing cell culture medium for $24 \mathrm{~h}$. Then, $T B X 2$-specific siRNA (si-TBX2) and negative control scrambled siRNA (si-NC) (GenePharma Co., Ltd, Suzhou, China) were administered with RiboFECTCP (Guangzhou RiboBio Co., Ltd., Guangzhou, China) reagent into cells at a confluence of approximately $70 \%$ according to the manufacturers' instructions and our previous study ${ }^{16}$. Then, the cells were incubated for $48 \mathrm{~h}$ at $37{ }^{\circ} \mathrm{C}$ in a $5 \% \mathrm{CO}_{2}$ incubator without changing the culture medium. The specific sequences of the siRNAs are shown in Supplementary Table 1.

Cell cycle assay. In brief, $1 \times 10^{5}$ cells were seeded in 6-well plates. After treatment with si-TBX2 or si-NC for $48 \mathrm{~h}$, the cell cycle distribution was determined using a Cell Cycle and Apoptosis Analysis Kit (Beyotime, Shanghai, China) and a flow cytometer (Beckman Coulter, Brea, CA, USA) according to the manufacturers' instructions. The data were processed by using MODFIT software (Verity Software House, Topsham, ME, USA). 
qRT-PCR assay. Total RNA was extracted using Tripure Isolation Reagent (Roche, Basel, Switzerland). cDNA was synthesized from the extracted RNA with a reverse transcription kit (Tiangen, Beijing, China) according to the instructions. Gene expression was quantified with a Mastercycler ep realplex system (Eppendorf, Hamburg, Germany) and the $2^{-\Delta \Delta \mathrm{Ct}}$ method with $\beta$-actin as the standard using the following protocol: $95^{\circ} \mathrm{C}$ for $3 \mathrm{~min} ; 40$ cycles at $95^{\circ} \mathrm{C}$ for $30 \mathrm{sec}, 60^{\circ} \mathrm{C}$ for $30 \mathrm{sec}$, and $72^{\circ} \mathrm{C}$ for $30 \mathrm{sec}$. All primers used are listed in Supplementary Table 1.

Cell proliferation assay. The cell proliferation was assayed using xCELLigence system (Roche Applied Science and ACEA Biosciences, Mannheim, Germany) as described previously with some modifications ${ }^{54,55}$. In brief, $50 \mu \mathrm{L}$ of cell culture media with si-TBX2 or si-NC at room temperature was added into each well of E-plate 16. Then, the E-plate 16 was connected to the system and checked in the cell culture incubator for proper electrical-contacts and the background impedance was measured. Meanwhile, the cells were resuspended in cell culture medium with siRNAs. $100 \mu \mathrm{L}$ of each cell suspension containing $5 \times 10^{3}$ cells was added to the $50 \mu \mathrm{L}$ medium containing si-TBX2 or si-NC on E-plate 96 in order to determine the optimum cell concentration. After 30 min incubation at room temperature, E-plate 96 was placed into the cell culture incubator. Finally, cell proliferation was monitored every $30 \mathrm{~min}$ for a period of up to $40 \mathrm{~h}$ via the incorporated sensor electrode arrays of the E-Plate 96. The electrical impedance was measured by the RTCA-integrated software of the xCELLigence system as a dimensionless parameter termed cell index.

Apoptosis detection. The apoptosis of cumulus cells was detected according to the instructions of the Annexin V-FITC Apoptosis Analysis Kit (Tianjin Sungene Biotech Co, Ltd, Tianjin, China). In brief, after treatment with si-TBX2 or si-NC, cells were treated with trypsin and washed twice with PBS. Then, the cells were centrifuged at $800 \times \mathrm{g}$ for $5 \mathrm{~min}$ at $4{ }^{\circ} \mathrm{C}$ and 3 times. Then, $100 \mu \mathrm{L}$ of PBS was added to each centrifuge tube, followed by incubation for 15 min with $5 \mu \mathrm{L}$ of FITC-labeled Annexin V solution and $5 \mu \mathrm{L}$ of PI $(20 \mu \mathrm{g} / \mathrm{mL})$ in the dark. Apoptotic cells and dead cells were distinguished by staining with PI and FITC-labeled Annexin V. The cell samples were then analyzed using a flow cytometer (Beckman Coulter).

Mitochondrial membrane potential (MMP) measurement. Briefly, the cell culture slides were placed into the 6-well plate before the cells were cultured. After treatment with si-TBX2 or si-NC, the culture medium was removed, and the cell slides were washed twice with PBS. Then, $1 \mathrm{~mL}$ of PBS containing $2 \mu \mathrm{M} \mathrm{JC}-1$ staining working solution was added to each of the wells, and the plates were incubated at $37^{\circ} \mathrm{C}$ for 30 min. After incubation, the culture slides were washed twice with PBS and mounted onto glass slides. The red and green fluorescence intensities were observed with a fluorescence microscope (Olympus, Shibuya, Japan). Image-Pro Plus software was used to analyze the red and green fluorescence and compare the ratio between the average optical densities in each sample. The relative fluorescence intensity level was measured as the average fluorescence intensity of individual cells (JC- $\left.1_{\text {red }} / \mathrm{JC}-1_{\text {green }}\right)$ 
from 3 independent experiments with 3 randomly selected observation images.

Determination of ROS levels. We first detected the level of ROS by microscopic fluorescence imaging. The cell culture slides were placed in a 6-well plate before the cells were cultured. After treatment with si-TBX2 or si-NC, the culture medium was removed, and the cell slides were washed twice with PBS. Subsequently, $1 \mathrm{~mL}$ DMEM/F12 culture medium containing $10 \mu \mathrm{M}$ DCFH-DA, (Beyotime) was added to each well of a 6-well plate. After incubation for $30 \mathrm{~min}$ in a $38.5^{\circ} \mathrm{C}$ incubator, the culture slides were washed 3 times with PBS and mounted onto glass slides. Finally, the fluorescence intensity of the cells was observed by a fluorescence microscope (Olympus). The level of ROS was assessed by comparing the fluorescence intensity among the different treatment groups. The relative fluorescence intensity level was measured as the average fluorescence intensity of individual cells from 3 independent experiments in 3 randomly selected fields.

Meanwhile, the changes in the ROS level were also detected by a Reactive Oxygen Species Assay Kit (Beyotime). Briefly, the siRNA treatments were the same as those described above. After washing and removing the cell culture medium, $10 \mu \mathrm{M} \mathrm{DCFH-}$ DA solution was added to each well of a six-well plate. After incubation for $30 \mathrm{~min}$ in a $38.5^{\circ} \mathrm{C}$ incubator, the samples were washed three times with PBS. The samples were harvested and then analyzed by flow cytometry (Beckman Coulter).

Immunofluorescence detection. The cell culture slides were placed into the 6-well plate before the cells were cultured. After treatment with si-TBX2 or si-NC, the culture medium was removed, and the cells were washed twice with PBS. After being fixed with $4 \%$ paraformaldehyde for $30 \mathrm{~min}$ and being washed three times with PBS, the cells were permeabilized with $0.3 \%$ Triton X-100 in PBS for 30 min and blocked in an incubator at $38.5{ }^{\circ} \mathrm{C}$ for $1 \mathrm{~h}$ in PBS containing 3\% BSA. Then, the cells were incubated at $4{ }^{\circ} \mathrm{C}$ with primary anti-LC3B antibody (Abcam, Cambridge, UK, \#ab48394, 1:200) overnight. Subsequently, after being washed three times with PBS, the cells were incubated with secondary antibody (Abcam, \#ab150077, 1:1000) at $38.5^{\circ} \mathrm{C}$ for $1 \mathrm{~h}$, washed three times and stained with Hoechst 33342 for $10 \mathrm{~min}$. Finally, after being washed 3 times with PBS, the culture slides were mounted onto glass slides. A Zeiss LSM 510 confocal microscope (Carl Zeiss, Jena, Germany) and Image-Pro Plus software were used to detect and analyze the images, respectively. The relative fluorescence intensity level was measured as the average fluorescence intensity of individual cells from 3 independent experiments in 3 randomly selected fields.

Cell expansion detection. The cells were cultured and treated with si-TBX2 or si-NC into the 6-well plates. After removing the culture medium, the middle area of the 6 -well plate was scraped with a cell-free knife, and serum-free DMEM/F12 medium was added for culture. The width of the cell scratches was detected at $0,12,24$, and $36 \mathrm{~h}$ through optical microscopy (Motic, Xiamen, Fujian, China). Image-Pro Plus software was used to calculate the average width of the scratch, which was used to determine cell expansion. 
Western blotting analysis. The cells were cultured and treated with si-TBX2 or si$\mathrm{NC}$ in 6-well plates as described above. After removing the culture medium, cell lysis buffer containing protease inhibitors (Beyotime) was added to the well and the samples were ultrasonicated for $60 \mathrm{~s}$ ( 3 times per sec) with an ultrasound transducer. After sonication, the cell sample mixtures were harvested and were placed on ice for $30 \mathrm{~min}$. Then, the mixtures were centrifuged for $60 \mathrm{~min}$ at $13,000 \times \mathrm{g}$, and the protein-containing supernatant was collected. Subsequently, protein samples were separated by SDSPAGE electrophoresis and then transferred to a $0.45-\mu \mathrm{m}$ PVDF membrane (Millipore, Bedford, MA, USA). Next, the membrane was transferred to 5\% BSA blocking solution and placed on an oscillator for $2 \mathrm{~h}$ at room temperature and then incubated with specific primary antibodies (anti-LC3B, Abcam, \#ab48394, 1:1,000; and anti-actin, Cell Signaling Technology, Beverly, MA, USA, \#4970, 1:1,000) overnight at $4{ }^{\circ} \mathrm{C}$. After being washed with TBST, the membranes were incubated with related horseradish peroxidase-conjugated secondary antibodies (Cell Signaling Technology, \#7074, 1:5000) at $37{ }^{\circ} \mathrm{C}$ for $1 \mathrm{~h}$. The membranes were then incubated in enhanced chemiluminescence reagents (Thermo, Waltham, MA, USA), and images were captured by a Tanon 5200 chemiluminescence imaging analyzer (Tanon Science \& Technology Co., Ltd., Shanghai, China). Finally, grayscale analysis was performed using ImagePro Plus software.

Statistical analysis. The results were obtained from three repeated independent experiments, and were expressed as mean \pm SD. Data obtained from two groups were compared using the Student's t-test. All statistical analyses were performed using SPSS version 22.0 (IBM, IL, USA) software. $\mathrm{P}<0.05$ and $\mathrm{P}<0.01$ were considered to indicate significant differences.

\section{References}

$1 \mathrm{Xu}, \mathrm{H}$. Y. et al. Treatment with acetyl-I-carnitine during in vitro maturation of buffalo oocytes improves oocyte quality and subsequent embryonic development. Theriogenology 118, 80-89, doi:10.1016/j.theriogenology.2018.05.033 (2018).

2 Keefe, D., Kumar, M. \& Kalmbach, K. Oocyte competency is the key to embryo potential. Fertility and sterility 103, 317-322, doi:10.1016/j.fertnstert.2014.12.115 (2015).

3 Dumesic, D. A., Meldrum, D. R., Katz-Jaffe, M. G., Krisher, R. L. \& Schoolcraft, W. B. Oocyte environment: follicular fluid and cumulus cells are critical for oocyte health. Fertility and sterility 103, 303-316, doi:10.1016/j.fertnstert.2014.11.015 (2015).

Petro, E. M. et al. Endocrine-disrupting chemicals in human follicular fluid impair in vitro 1033, doi:10.1093/humrep/der448 (2012).

5 Lin, T., Oqani, R. K., Lee, J. E., Shin, H. Y. \& Jin, D. I. Coculture with good-quality COCs enhances the maturation and development rates of poor-quality COCs. Theriogenology 85, 396-407, doi:10.1016/j.theriogenology.2015.09.001 (2016). 
cell function and oocyte quality. Human reproduction update 14, 159-177, doi:10.1093/humupd/dmm040 (2008).

7 Shaeib, F. et al. The Defensive Role of Cumulus Cells Against Reactive Oxygen Species Insult in Metaphase II Mouse Oocytes. Reprod Sci 23, 498-507, doi:10.1177/1933719115607993 (2016).

8 Cetica, P. D., Pintos, L. N., Dalvit, G. C. \& Beconi, M. T. Effect of lactate dehydrogenase activity and isoenzyme localization in bovine oocytes and utilization of oxidative substrates on in vitro maturation. Theriogenology 51, 541-550, doi:10.1016/s0093691x(99)00008-4 (1999).

9 Cetica, P. D., Pintos, L. N., Dalvit, G. C. \& Beconi, M. T. Antioxidant enzyme activity and oxidative stress in bovine oocyte in vitro maturation. IUBMB life 51, 57-64, doi:10.1080/15216540119253 (2001).

10 Oyewole, A. O. \& Birch-Machin, M. A. Mitochondria-targeted antioxidants. FASEB journal: official publication of the Federation of American Societies for Experimental Biology 29, 4766-4771, doi:10.1096/fj.15-275404 (2015).

11 Scherz-Shouval, R. et al. Reactive oxygen species are essential for autophagy and specifically regulate the activity of Atg4. The EMBO journal 26, 1749-1760, doi:10.1038/sj.emboj.7601623 (2007).

12 Kulsoom, B. et al. Bax, $\mathrm{Bcl}-2$, and $\mathrm{Bax} / \mathrm{BCl}-2$ as prognostic markers in acute myeloid leukemia: are we ready for $\mathrm{Bcl}$-2-directed therapy? Cancer management and research 10 , 403-416, doi:10.2147/cmar.S154608 (2018).

13 Lossi, L., Castagna, C. \& Merighi, A. Caspase-3 Mediated Cell Death in the Normal Development of the Mammalian Cerebellum. International journal of molecular sciences 19, doi:10.3390/ijms19123999 (2018).

14 Zhao, T., Fu, Y., Sun, H. \& Liu, X. Ligustrazine suppresses neuron apoptosis via the Bax/Bcl2 and caspase- 3 pathway in PC12 cells and in rats with vascular dementia. IUBMB life 70 , 60-70, doi:10.1002/iub.1704 (2018).

15 Scherz-Shouval, R. \& Elazar, Z. ROS, mitochondria and the regulation of autophagy.

$16 \mathrm{Fu}, \mathrm{X} . \mathrm{H}$. et al. COL1A1 affects apoptosis by regulating oxidative stress and autophagy in bovine cumulus cells. Theriogenology 139, 81-89, doi:10.1016/j.theriogenology.2019.07.024 (2019).

17 Huang, Z. \& Wells, D. The human oocyte and cumulus cells relationship: new insights from the cumulus cell transcriptome. Molecular human reproduction 16, 715-725 (2010).

18 Adriaenssens, T. et al. Cumulus cell gene expression is associated with oocyte developmental quality and influenced by patient and treatment characteristics. Human reproduction 25, 1259-1270 (2010).

19 Chapman, D. L. et al. Expression of the T-box family genes, Tbx1-Tbx5, during early mouse development. Developmental dynamics : an official publication of the American Association of Anatomists 206, 379-390, doi:10.1002/(sici)10970177(199608)206:4<379::Aid-aja4>3.0.Co;2-f (1996).

20 Teegala, S., Chauhan, R., Lei, E. \& Weinstein, D. C. Tbx2 is required for the suppression of mesendoderm during early Xenopus development. Developmental dynamics : an official publication of the American Association of Anatomists 247, 903-913, 
doi:10.1002/dvdy.24633 (2018).

1 Abrahams, A., Parker, M. I. \& Prince, S. The T-box transcription factor Tbx2: its role in development and possible implication in cancer. IUBMB life 62, 92-102, doi:10.1002/iub.275 (2010).

22 Peres, J. et al. The Highly Homologous T-Box Transcription Factors, TBX2 and TBX3, Have Distinct Roles in the Oncogenic Process. Genes \& cancer 1, 272-282, doi:10.1177/1947601910365160 (2010).

23 Jacobs, J. J. et al. Senescence bypass screen identifies TBX2, which represses Cdkn2a (p19(ARF)) and is amplified in a subset of human breast cancers. Nature genetics 26, 291299, doi:10.1038/81583 (2000).

24 Liu, X. et al. TBX2 overexpression promotes proliferation and invasion through epithelialmesenchymal transition and ERK signaling pathway. Exp Ther Med 17, 723-729, doi:10.3892/etm.2018.7028 (2019).

25 Crawford, N. T. et al. TBX2 interacts with heterochromatin protein 1 to recruit a novel repression complex to EGR1-targeted promoters to drive the proliferation of breast cancer cells. Oncogene 38, 5971-5986, doi:10.1038/s41388-019-0853-z (2019).

26 Pietenpol, J. A. \& Stewart, Z. A. Cell cycle checkpoint signaling: cell cycle arrest versus apoptosis. Toxicology 181-182, 475-481, doi:10.1016/s0300-483x(02)00460-2 (2002).

27 Ye, K. et al. Effect of norcantharidin on the proliferation, apoptosis, and cell cycle of human mesangial cells. Renal failure 39, 458-464, doi:10.1080/0886022x.2017.1308257 (2017).

Yi, F., Du, J., Ni, W. \& Liu, W. Tbx2 confers poor prognosis in glioblastoma and promotes temozolomide resistance with change of mitochondrial dynamics. Onco Targets Ther 10 , 1059-1069, doi:10.2147/OTT.S124012 (2017).

29 Pan, L. et al. Microphthalmia-associated transcription factor/T-box factor-2 axis acts through Cyclin D1 to regulate melanocyte proliferation. Cell Prolif 48, 631-642, doi:10.1111/cpr.12227 (2015).

30 Ohtsubo, M., Theodoras, A. M., Schumacher, J., Roberts, J. M. \& Pagano, M. Human cyclin E, a nuclear protein essential for the G1-to-S phase transition. Mol Cell Biol 15, 26122624, doi:10.1128/mcb.15.5.2612 (1995).

31 Ferguson, R. L. \& Maller, J. L. Centrosomal localization of cyclin E-Cdk2 is required for initiation of DNA synthesis. Curr Bio/ 20, 856-860, doi:10.1016/j.cub.2010.03.028 (2010).

Barr, A. R. \& Gergely, F. Aurora-A: the maker and breaker of spindle poles. J Cell Sci 120, 2987-2996, doi:10.1242/jcs.013136 (2007).

33 Ito, M. Factors controlling cyclin B expression. Plant Mol Biol 43, 677-690, doi:10.1023/a:1006336005587 (2000).

34 Sherr, C. J. \& Roberts, J. M. Living with or without cyclins and cyclin-dependent kinases. Genes Dev 18, 2699-2711, doi:10.1101/gad.1256504 (2004).

35 Bai, J., Li, Y. \& Zhang, G. Cell cycle regulation and anticancer drug discovery. Cancer Biol Med 14, 348-362, doi:10.20892/j.issn.2095-3941.2017.0033 (2017).

36 Mandal, S., Lindgren, A. G., Srivastava, A. S., Clark, A. T. \& Banerjee, U. Mitochondrial function controls proliferation and early differentiation potential of embryonic stem cells. Stem Cells 29, 486-495, doi:10.1002/stem.590 (2011).

$37 \mathrm{Fu}, \mathrm{X} . \mathrm{H}$. et al. Dual-specificity phosphatase 1 regulates cell cycle progression and apoptosis in cumulus cells by affecting mitochondrial function, oxidative stress, and 
autophagy. Am J Physio/ Cell Physio/ 317, C1183-C1193, doi:10.1152/ajpcell.00012.2019 (2019).

38 Atsumi, T., Tonosaki, K. \& Fujisawa, S. Induction of early apoptosis and ROS-generation activity in human gingival fibroblasts (HGF) and human submandibular gland carcinoma (HSG) cells treated with curcumin. Archives of Oral Biology 51, 913-921 (2006).

39 Yi, F., Du, J., Ni, W. \& Liu, W. Tbx2 confers poor prognosis in glioblastoma and promotes temozolomide resistance with change of mitochondrial dynamics. OncoTargets and therapy 10, 1059 (2017).

40 Zorov, D. B., Juhaszova, M. \& Sollott, S. J. Mitochondrial reactive oxygen species (ROS) and ROS-induced ROS release. Physiological reviews 94, 909-950, doi:10.1152/physrev.00026.2013 (2014).

41 Cadenas, S. Mitochondrial uncoupling, ROS generation and cardioprotection. Biochimica et biophysica acta. Bioenergetics 1859, 940-950, doi:10.1016/j.bbabio.2018.05.019 (2018).

42 Wang, X. et al. Betulinic Acid Induces Apoptosis in Differentiated PC12 Cells Via ROSMediated Mitochondrial Pathway. Neurochemical research 42, 1130-1140, doi:10.1007/s11064-016-2147-y (2017).

43 Twig, G. et al. Fission and selective fusion govern mitochondrial segregation and elimination by autophagy. EMBO J27, 433-446, doi:10.1038/sj.emboj.7601963 (2008).

44 Lee, E. et al. Autophagy is essential for cardiac morphogenesis during vertebrate development. Autophagy 10, 572-587, doi:10.4161/auto.27649 (2014).

45 Fang, Y. et al. Melatonin-induced demethylation of antioxidant genes increases antioxidant capacity through $\mathrm{ROR} \alpha$ in cumulus cells of prepubertal lambs. Free radical biology \& medicine 131, 173-183, doi:10.1016/j.freeradbiomed.2018.11.027 (2019).

46 Kahraman, S. et al. Is there a correlation between follicle size and gene expression in cumulus cells and is gene expression an indicator of embryo development? Reproductive biology and endocrinology : RB\&E 16, 69, doi:10.1186/s12958-018-0388-0 (2018).

47 Su, Y. Q. et al. Oocyte regulation of metabolic cooperativity between mouse cumulus cells and oocytes: BMP15 and GDF9 control cholesterol biosynthesis in cumulus cells. Development 135, 111-121, doi:10.1242/dev.009068 (2008).

48 Payne, A. H. \& Hales, D. B. Overview of steroidogenic enzymes in the pathway from cholesterol to active steroid hormones. Endocr Rev 25, 947-970, doi:10.1210/er.20030030 (2004).

49 Su, Y. Q., Sugiura, K. \& Eppig, J. J. Mouse oocyte control of granulosa cell development and function: paracrine regulation of cumulus cell metabolism. Seminars in reproductive medicine 27, 32-42, doi:10.1055/s-0028-1108008 (2009).

50 Sugiura, K. et al. Oocyte-derived BMP15 and FGFs cooperate to promote glycolysis in cumulus cells. Development 134, 2593-2603, doi:10.1242/dev.006882 (2007).

51 Miller, W. L. Steroidogenesis: Unanswered Questions. Trends Endocrinol Metab 28, 771 793, doi:10.1016/j.tem.2017.09.002 (2017).

52 Watanabe, H., Hirai, S., Tateno, H. \& Fukui, Y. Variation of cholesterol contents in porcine cumulus-oocyte complexes is a key factor in regulation of fertilizing capacity. Theriogenology 79, 680-686, doi:10.1016/j.theriogenology.2012.11.024 (2013).

53 Bunel, A. et al. Cumulus cell gene expression associated with pre-ovulatory acquisition of developmental competence in bovine oocytes. Reprod Fertil Dev 26, 855-865, 
doi:10.1071/RD13061 (2014).

54 Urcan, E. et al. Real-time xCELLigence impedance analysis of the cytotoxicity of dental composite components on human gingival fibroblasts. Dent Mater 26, 51-58, doi:10.1016/j.dental.2009.08.007 (2010).

55 Bird, C. \& Kirstein, S. Real-time, label-free monitoring of cellular invasion and migration with the XCELLigence system. Nature methods 6, v-vi (2009).

\section{Figure legend}

Fig. 1. TBX2 inhibition affects the cell cycle, proliferation, and apoptosis.

(A) The percentage of cells in each phase of the cell cycle with or without $T B X 2$ inhibition. (B) Differential expression of $C D K 1, C D K 2, C D K 4$, and $C D K 6$ in the si-NC and $T B X 2$ inhibition groups. (C) Cell index in the si-NC and $T B X 2$ inhibition groups. (D) Percentage of apoptotic cells in the si-NC and $T B X 2$ inhibition groups. Significant differences are represented with ** $(\mathrm{P}<0.01)$. (E) Changes in $B A X / B C L 2$ mRNA levels. Significant differences are represented with $* *(\mathrm{P}<0.01)$.

\section{Fig. 2. ROS levels in the si-TBX2 and si-NC groups.}

(A) Representative fluorescence images of DCFH staining of cumulus cells. (B) Differences in DCFH levels between the si-NC and TBX2-inhibited groups. (C) ROS levels in the si-NC and TBX2-inhibited groups as detected by flow cytometry. Significant differences are represented with $* *(\mathrm{P}<0.01)$.

Fig. 3. Changes in mitochondrial membrane potential and ATP levels after inhibition of $\mathbf{T B X} 2$.

(A) Representative fluorescence images of JC-1 staining in the $T B X 2$ inhibition group and si-NC group. (B) Relative fluorescence intensity of $\mathrm{JC}-1_{\text {red }} / \mathrm{JC}-1_{\text {green }}$ in the si-NC and TBX2-inhibited groups. (C) Relative ATP levels in the si-NC and TBX2-inhibited groups. Significant differences are represented with ** $(\mathrm{P}<0.01)$.

Fig. 4. Autophagy level changes after inhibition of $T B X 2$.

(A) Representative $L C 3 B$ staining images in the si-NC and $T B X 2$-inhibited groups analyzed by immunofluorescence. (B) Protein expression of $L C 3 B$ in the si-NC (left side) and si-TBX2 groups (right side) come from the same gel. Significant differences are represented with ** $(\mathrm{P}<0.01)$.

\section{Fig. 5. Inhibition of $T B X 2$ reduces cumulus cell expansion and inhibits cholesterol} synthesis.

(A) Representative images of cumulus cell expansion at $0,12,24$, and $36 \mathrm{~h}$ with or without $T B X 2$ inhibition. (B) Compared with the si-NC group, the $T B X 2$ inhibition group showed significantly lower cell expansion levels over time, especially at $24 \mathrm{~h}$ 
573 and 36 h. (C) Relative PTGS2, PTX3, and HAS2 expression changes between the si-NC 574 and $T B X 2$-inhibited groups. (D) The relative level of cholesterol was decreased in the $575 T B X 2$-inhibited group compared with the si-NC group. Significant differences are 576 represented with ** $(\mathrm{P}<0.01)$. 
Figures

A

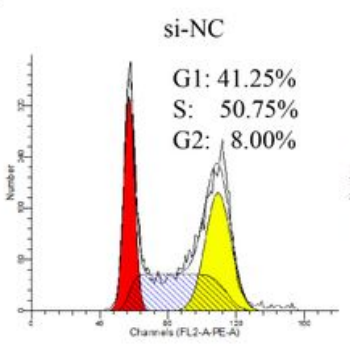

$\mathrm{C}$

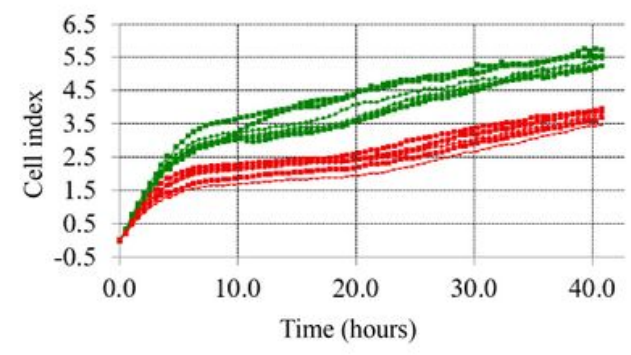

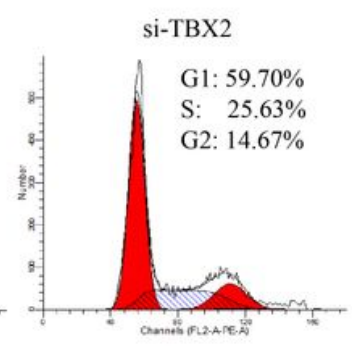

$\mathrm{D}$
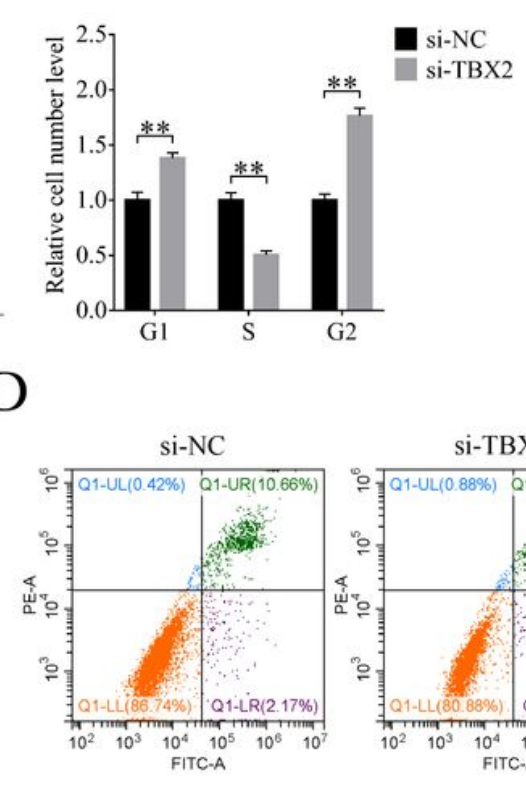

D
B

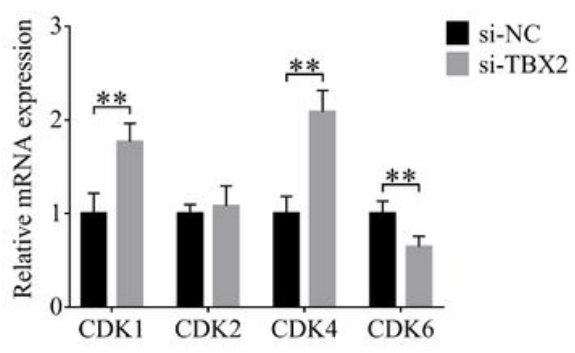

$\mathrm{E}$

Figure 1

TBX2 inhibition affects the cell cycle, proliferation, and apoptosis. (A) The percentage of cells in each phase of the cell cycle with or without TBX2 inhibition. (B) Differential expression of CDK1, CDK2, CDK4, and CDK6 in the si-NC and TBX2 inhibition groups. (C) Cell index in the si-NC and TBX2 inhibition groups. (D) Percentage of apoptotic cells in the si-NC and TBX2 inhibition groups. Significant differences are represented with ** $(P<0.01)$. (E) Changes in BAX/BCL2 mRNA levels. Significant differences are represented with $* *(P<0.01)$. 
A
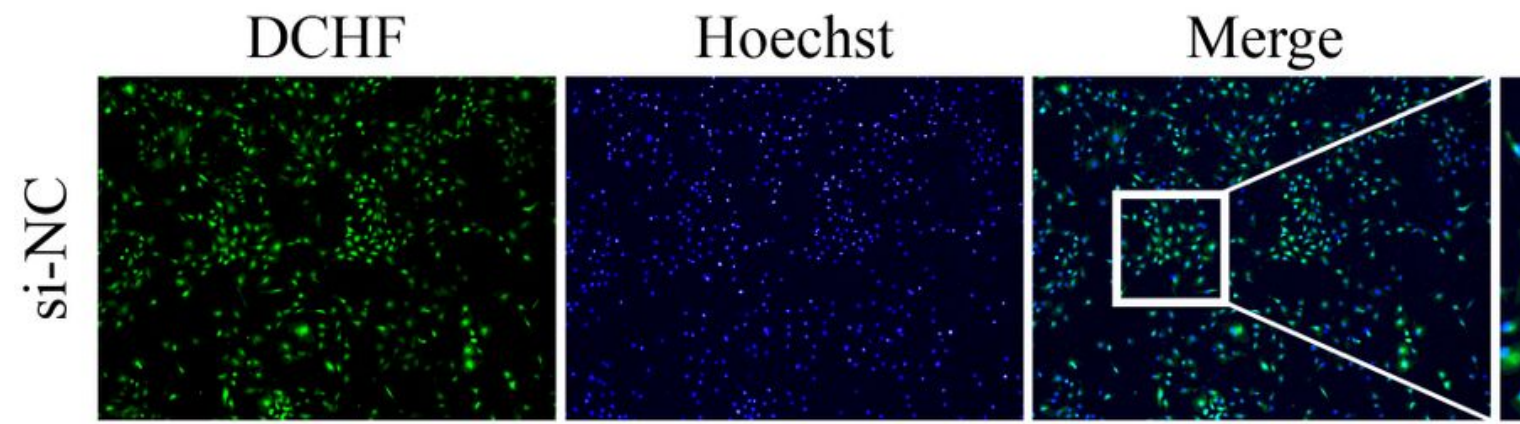

Enlarge
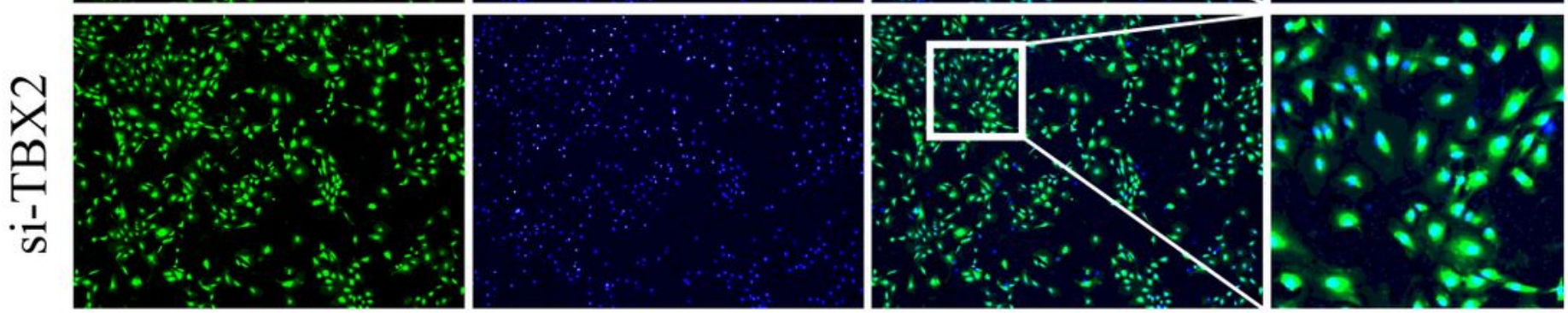

$\mathrm{B}$
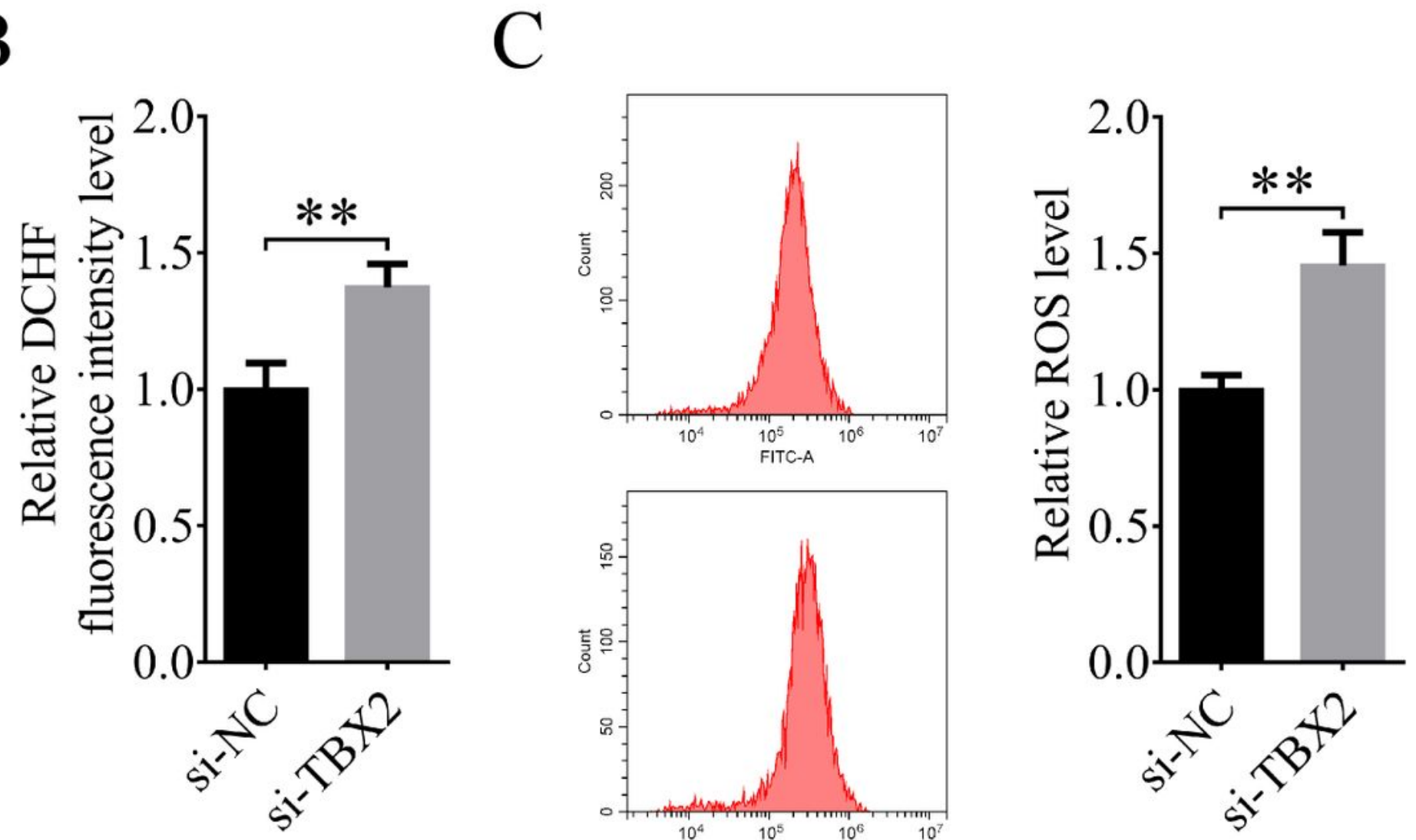

Figure 2

ROS levels in the si-TBX2 and si-NC groups. (A) Representative fluorescence images of DCFH staining of cumulus cells. (B) Differences in DCFH levels between the si-NC and TBX2-inhibited groups. (C) ROS levels in the si-NC and TBX2-inhibited groups as detected by flow cytometry. Significant differences are represented with $* *(P<0.01)$. 
A

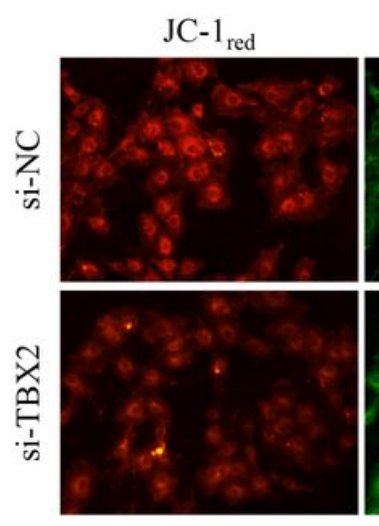

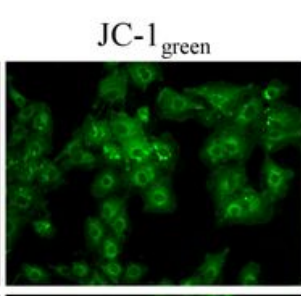
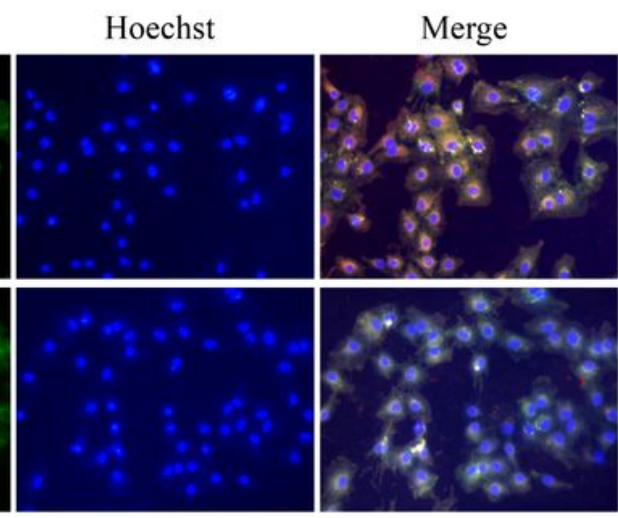

$\mathrm{B}$

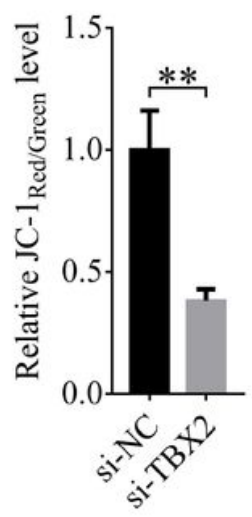

$\mathrm{C}$

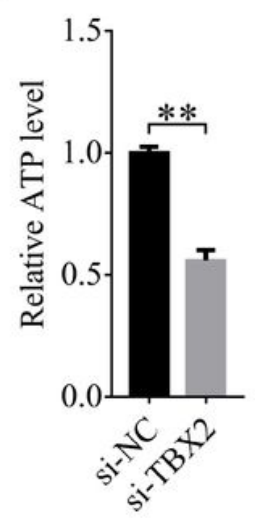

\section{Figure 3}

Changes in mitochondrial membrane potential and ATP levels after inhibition of TBX2. (A) Representative fluorescence images of JC-1 staining in the TBX2 inhibition group and si-NC group. (B) Relative fluorescence intensity of JC-1 red/JC-1 green in the si-NC and TBX2-inhibited groups. (C) Relative ATP levels in the si-NC and TBX2-inhibited groups. Significant differences are represented with $* *(P<0.01)$.

A

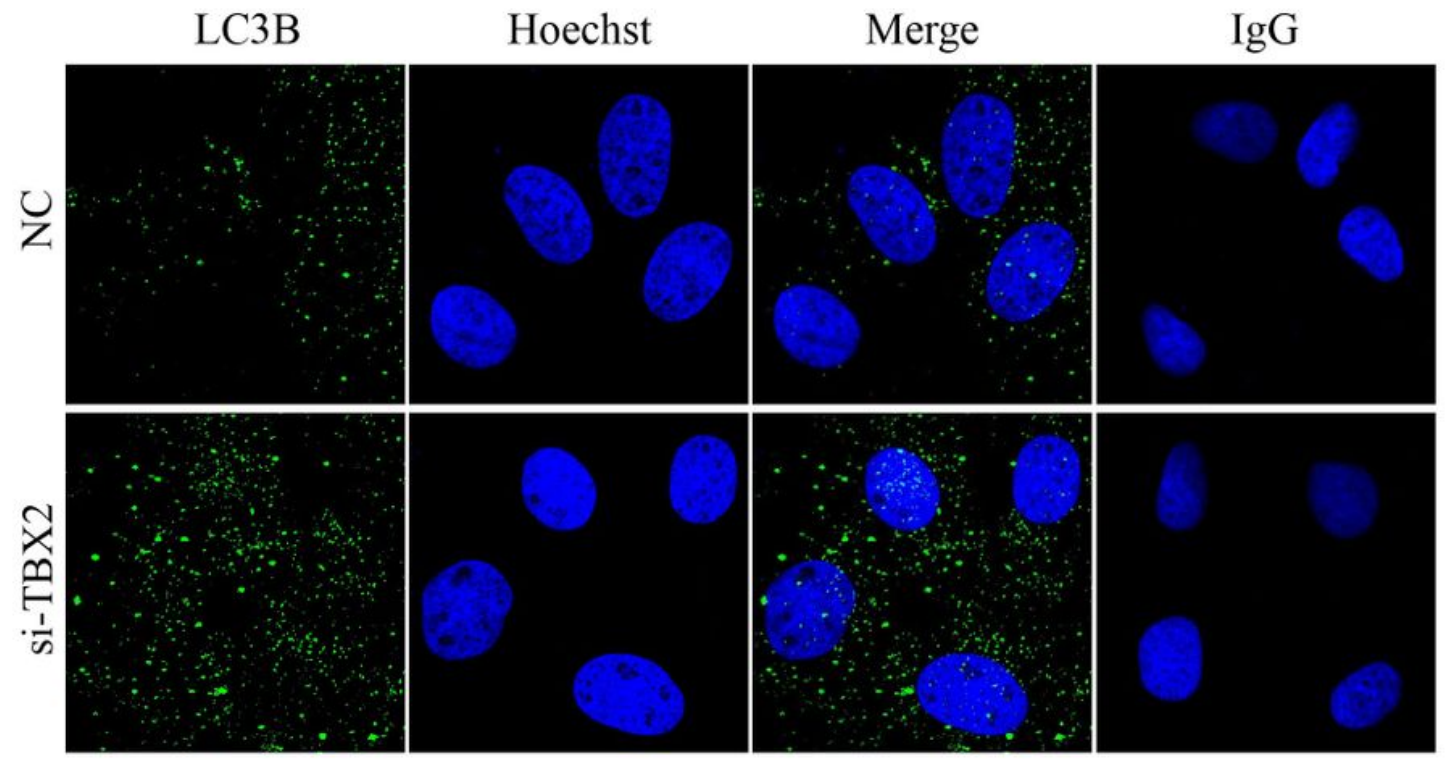

B

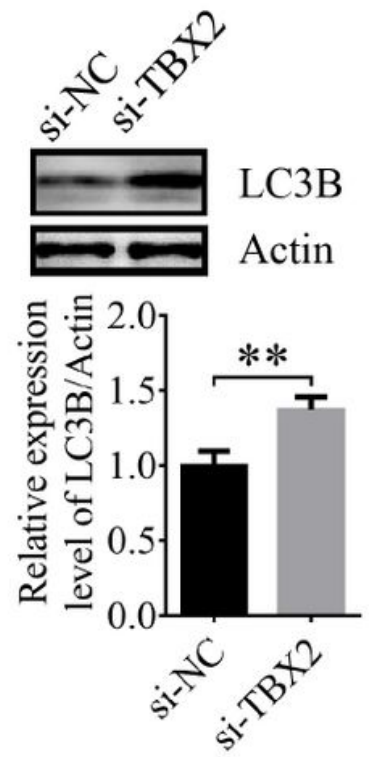

Figure 4

Autophagy level changes after inhibition of TBX2. (A) Representative LC3B staining images in the si-NC and TBX2-inhibited groups analyzed by immunofluorescence. (B) Protein expression of LC3B in the si-NC (left side) and si-TBX2 groups (right side) come from the same gel. Significant differences are represented with $* *(P<0.01)$. 
A

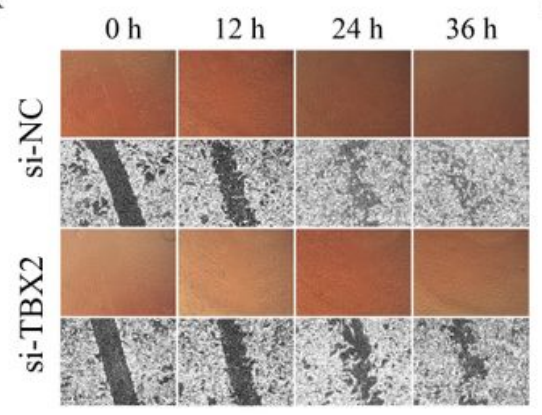

$\mathrm{B}$

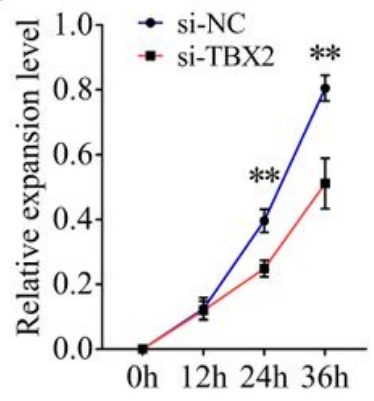

$\mathrm{C}$

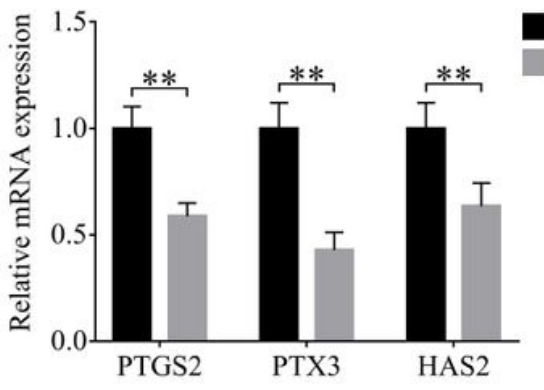

$\mathrm{D}$

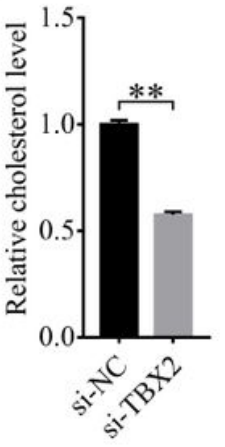

Figure 5

Inhibition of TBX2 reduces cumulus cell expansion and inhibits cholesterol synthesis. (A) Representative images of cumulus cell expansion at $0,12,24$, and $36 \mathrm{~h}$ with or without TBX2 inhibition. (B) Compared with the si-NC group, the TBX2 inhibition group showed significantly lower cell expansion levels over time, especially at $24 \mathrm{~h}$ and $36 \mathrm{~h}$. (C) Relative PTGS2, PTX3, and HAS2 expression changes between the si-NC and TBX2-inhibited groups. (D) The relative level of cholesterol was decreased in the TBX2-inhibited group compared with the si-NC group. Significant differences are represented with ** $(P<0.01)$.

\section{Supplementary Files}

This is a list of supplementary files associated with this preprint. Click to download.

- SupplementaryMaterial.docx 